\title{
TRANSARTICULAR NAILING FOR FRACTURES OF THE FEMORAL NECK*
}

\author{
A Preliminary Report \\ Leo Jarry, Montreal, Canada \\ From the Orthopaedic Department, Verdun General Hospital, Montreal
}

Research on avascular necrosis, creeping substitution, mechanics and circulation in relation to the femoral head has led to a better understanding of fracture at the hip. Despite this better knowledge, it has been impossible to solve the problem of the high incidence of avascular necrosis and pseudarthrosis. It seems that a deeper comprehension of the hip problem has given us a " defeatist attitude " (Dickson 1953) in the matter of these complications to the extent of forsaking osteosynthesis and sacrificing the femoral head for a prosthesis.

However, advances such as improvement of fixation material and surgical techniques have diminished the incidence of complications. Undoubtedly, the overreduced position (McElvenny 1957) by preventing shearing stresses at the fracture site and by fixing of the femoral head in valgus contributes to the attainment of osseous union. This is especially so if, with diaphysial fixation, the nail penetrates deeply and rests on the calcar femoralis.

One would not easily recognise in transarticular nailing a basis for a contribution to the solution of the problem, because the procedure is insulting both to the joint and to the mind. Nevertheless, many transarticular fixations have been done because of technical error, and were often followed by bony union (Eaton 1956). We now believe that this unorthodox procedure might well have some advantages. It is presented as an alternative technique to replacement by prosthesis for use in the aged.

\section{PREVIOUS EXPERIENCE}

In 1935 Böhler did a transarticular fixation in a case of pseudarthrosis. Because the femoral head fragment was too small the long nail was purposely driven 20 millimetres into the acetabulum. The result in this case was favourable. Böhler added, without giving further details, that he had nailed the heads in four other cases with similar small fragments and that necrosis of the femoral head developed in every patient.

In $1939 \mathrm{King}$ reported a case of pseudarthrosis of the femoral neck treated by extraarticular osteosynthesis and a cortical bone graft. The Smith-Petersen nail was introduced intentionally into the free cavity of the acetabulum. It was then shown that the range of hip movement was full before and after removal of the nail. The hip was painless after two years. This procedure seems to have been limited to the one particular case.

\section{MATERIAL AND TECHNIQUE}

There were forty-four patients with fractures of the neck of the femur, excluding patients with impacted fractures. Ages varied from sixty-three to eighty-nine. In thirty-seven cases the guide wires were introduced percutaneously; in the other seven the introduction was done by the open method. The percutaneous approach minimises trauma and reduces the time of operation.

The operation was performed on a standard surgical table with a radiography tunnel. Leadbetter's method of reduction was used. The limb was firmly secured to the table with adhesive tape in order to prevent any movement of the hip joint which could kink the guide wire after introduction. The leg was held in 20 degrees of abduction outside the table, the

* Read in part at the annual meeting of the Canadian Orthopaedic Association, St Andrews-by-the-Sea, N.B., June 1961 . 
knee being flexed to 150 degrees with 25 degrees of medial rotation of the hip so as to place the long axis of the femoral neck in the horizontal plane. The femoral neck occupies the anterior two-thirds of the trochanter.

Two Kirschner wires one thirty-second inch thick were introduced antero-posteriorly, one above and one below the femoral neck and in contact with the latter. The guide wires were then introduced.

The guide wire three thirty-seconds of an inch thick was introduced at a point where an imaginary horizontal line drawn from the inferior border of the symphysis pubis crossed the lateral femoral cortex. It was pointed in a horizontal plane to aim between the two Kirschner wires that defined the femoral neck (Fig. 1). The guide wire should form an angle of about 130 degrees with the femoral shaft.

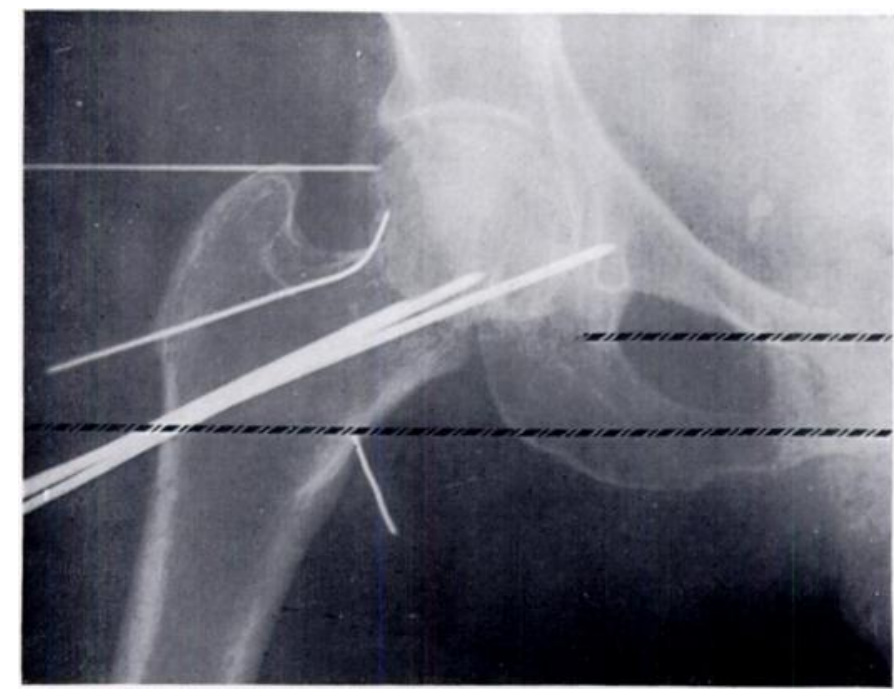

FIG. 1

Radiograph showing technique for introduction of guide wires. Note the Kirschner wires placed above and below the femoral neck, and the horizontal line at the level of the lower border of the symphysis pubis. indicating the point for introduction of guide wire.

When the guide wire encountered the resistance of the head and the acetabular wall a radiograph was taken to show the adequacy of reduction and the position of the wire. The guide wire might be central or eccentric, but care was taken to ensure that it did not lie in the weight-bearing area of the head and acetabulum - that is, beyond the summit at the junction of the upper two quadrants.

After the guide was secured deeply to prevent displacement of the head the SmithPetersen nail was introduced. When the percutaneous approach was used the nail was introduced through an incision one and a half inches long contiguous to the guide wire.

The length of the nail was determined by subtraction from the total length of the guide wire of the amount projecting from the cortex when the point was at the articular surface. To this length a maximum of three-quarters of an inch was added to correspond to the thickness of the acetabular wall. It must be remembered that the latter is not of uniform thickness.

At the end of the operation there was always adequate fixation of the hip with the limb in medial rotation. This fixation lasted for a time dependent on how much the patient moved in bed. Usually, limited movement was noticeable after three to five weeks. The nail might penetrate completely or partially into the acetabular floor to produce either a " tight locking effect" (Figs. 2 and 3) or a "loose locking effect" (Figs. 4 and 5).

VOL. 46 B, NO. 4, NOVEMBER 1964 


\section{CARE AFTER OPERATION}

The patients were divided into two groups-those unfit for convalescence in bed and those fit for convalescence in bed.

For the first group the wheel chair was used immediately after operation. These patients should be taught to use it in order to prevent weight bearing and to mobilise the hip when moving from bed to chair.

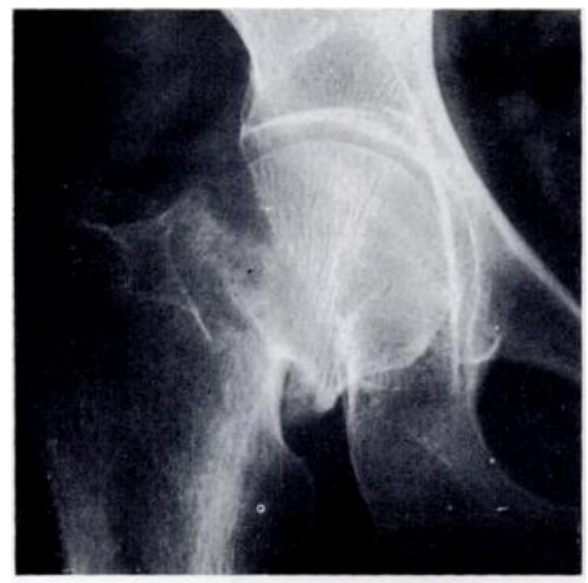

Fig. 2

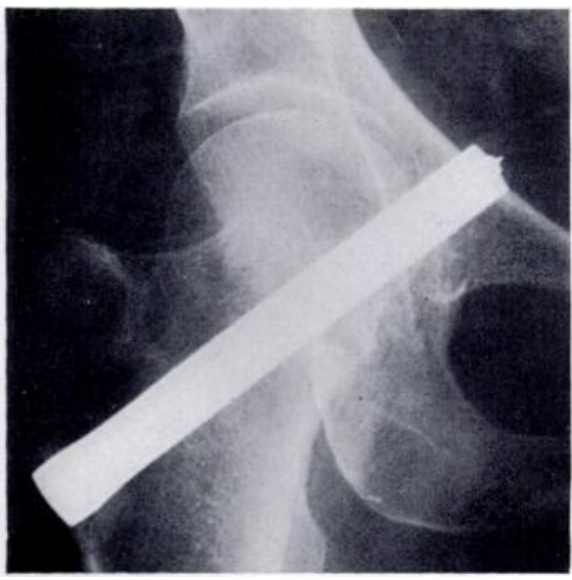

FIG. 3

Fracture of femoral neck (Pauwels' type 3) treated by transarticular nailing, with " tight locking" effect. Figure 2-Radiograph showing fracture before reduction. Figure 3-After reduction and nailing. Note the valgus position with rotation of head and deep penetration of nail.

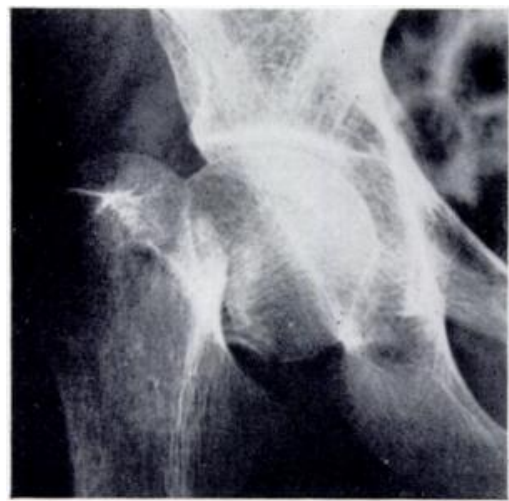

FIG. 4

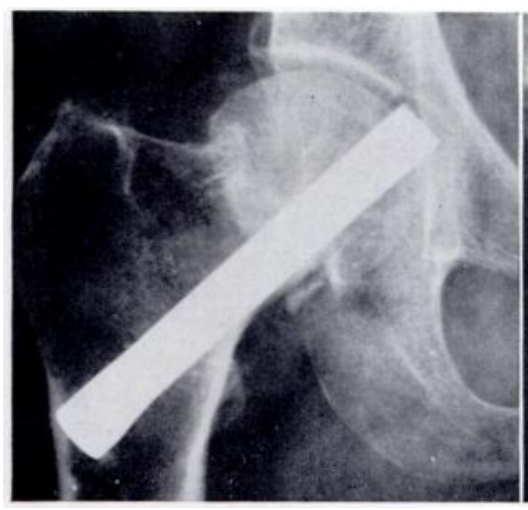

FiG. 5

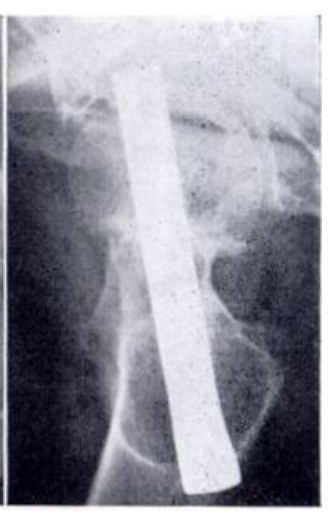

Subcapital fracture treated by transarticular nailing, with " loose locking" effect. Figure 4-Radiograph showing fracture before reduction. Figure 5-Antero-posterior and (right) lateral radiographs showing position after reduction and nailing. The position of the nail in the posterior-inferior part of the neck contributes to stability. The nail has only slightly penetrated the acetabulum, to produce the "loose locking " effect."

Patients in the second group were kept in bed for ninety days. The patients sat in bed and on the edge of the bed, and were active in bed. They were forbidden to make any effort wha tever to use the affected hip. When they lay supine in bed a cushion was placed under the knee, which was thus kept flexed at about 150 degrees. No exercise of the quadriceps muscles was allowed, and crossing the legs was avoided (Massie 1958). The word "fragile" was painted with methylene blue on the affected thigh, as advised by Crawford (1960) in the treatment of impacted fractures. 
After sixty days, active mobilisation by exercise of the quadriceps, elevation of the limb without effort and gradual flexion and extension of the hip were started.

After ninety days the patient was permitted to walk with a walker or crutches. Elevation of the limb with extended knee was not allowed. Adduction movements were not advised.

TABLE I

Results of Transarticular Nailing: Thirty-eight Patients (Excluding five who died within six months of operation and one who could not be traced)

\begin{tabular}{|l|c|c|c|c|c|}
\hline & $\begin{array}{c}\text { Number of } \\
\text { patients }\end{array}$ & $\begin{array}{c}\text { Diaphysial } \\
\text { fixation }\end{array}$ & Pseudarthrosis & $\begin{array}{c}\text { Posterior } \\
\text { rotation } \\
\text { of head }\end{array}$ & $\begin{array}{c}\text { Delayed } \\
\text { union after } \\
\text { one year }\end{array}$ \\
\hline Follow-up 1-6 years $\cdot$ & 22 & 2 & 1 & 4 & 2 \\
\hline Follow-up less than 1 year & 16 & 2 & - & - & - \\
\hline Total .. & 38 & - & - & - & - \\
\hline
\end{tabular}

If the head was in valgus the patient was warned particularly to abstain from any adduction movement so as to prevent tension of the ligamentum teres and its foveolar artery.

Between the fifth and the sixth months the patient was allowed gradually to bear weight. At the sixth month he might discard the walker or crutches and use a stick until at least the twelfth month. For a year after that, weight had to be borne on the unaffected limb only when the patient climbed stairs. If the nail protrudes distally, because of penetration of the neck into the femoral head, it must not be removed hastily. A protruding nail must be considered as protection, since it often prevents extensive movement.

\section{RESULTS}

Sixteen patients whose progress had been observed for less than a year were excluded from the analysis. Of the remaining twenty-eight, five are dead and one could not be traced. Thus there are twenty-two patients with a follow-up of from twelve months to six years. There was one case of pseudarthrosis; two of delayed union; and four of retroversion of the head (Table I). The neck of the femur was often resorbed. In no case was there disengagement of the nail. We never encountered necrosis of the femoral head at the tip of the nail (Watson-Jones 1955) or osteitis around the nail (Leira 1946). As to avascular necrosis, our observations are confined to ten cases with a follow-up of from two to six years. Among these there has been no instance of collapse caused by avascular necrosis.

Because the head is firmly secured in the cotyloid cavity when the nail is being hammered, the damage inflicted by the nail on the head is very localised and does not affect the surrounding area (Fig. 6).

FIG. 6

Specimen showing the effect of transarticular nailing on the femoral head. This specimen was obtained at necropsy twelve days after operation for a subcapital fracture of the femur. There was comminution of the back of the femoral neck. Note how the nail has cleanly penetrated the femoral head; one flange of the nail is seen through the comminuted area of the back of the femoral neck.

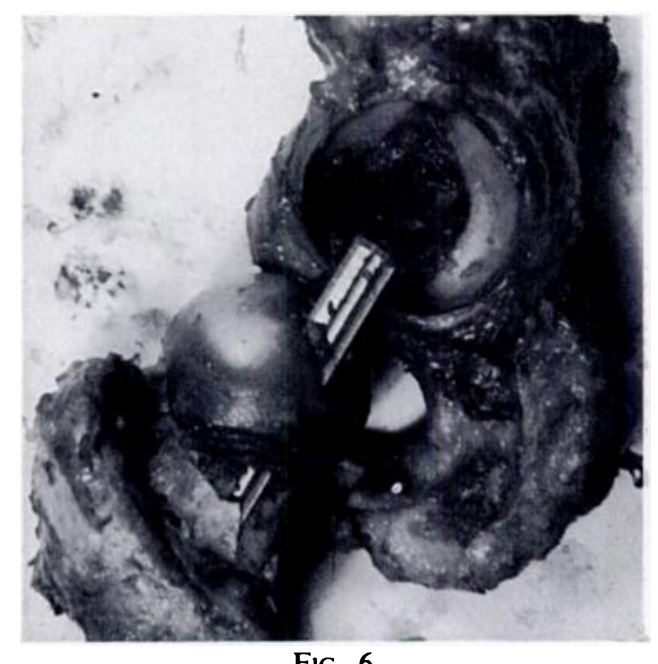

VOL. 46 B, NO. 4, NOVEMBER 1964 


\section{DISCUSSION}

Tolerance and intolerance of transarticular nailing in relation to the activity of the hip-A nail that prevents movement of the joint causes a painless erosion around its tip. If the nail penetrates the acetabular floor, a shell of new bone usually develops around its tip (Figs. 5 , 7 and 8). Transarticular fixation causes a decrease in all movements, particularly in abduction and in adduction, especially if the nail is located high in the acetabulum (Figs. 9 and 10).

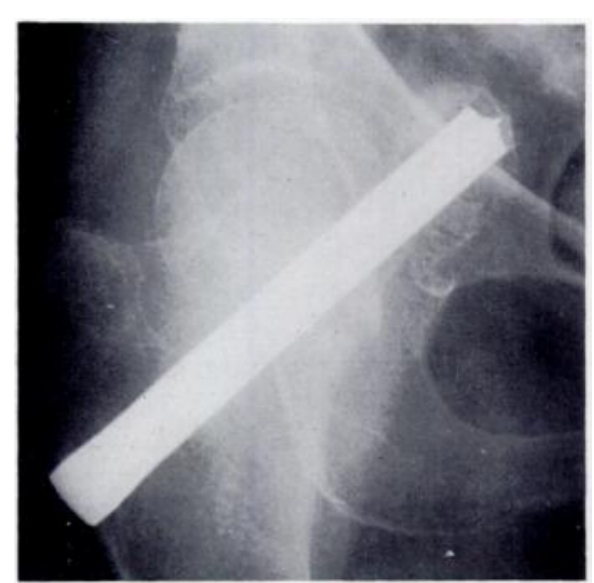

Fig. 7

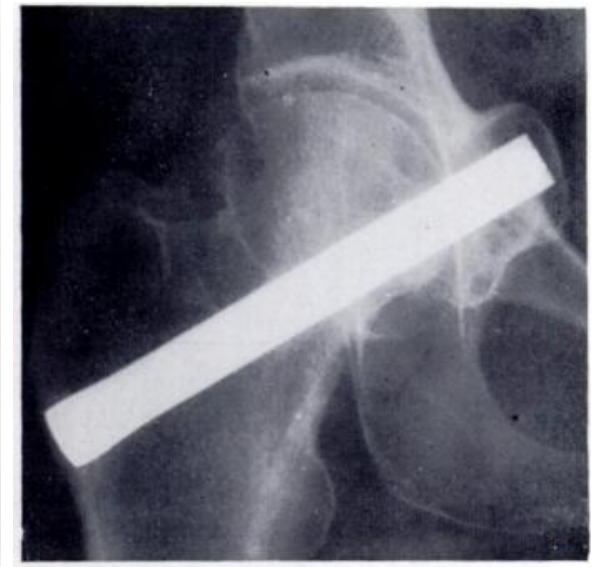

Fig. 8

Reactions to transarticular nailing. Figure 7-Antero-posterior radiograph of hip ninety days after nailing. Note the bony shell around the tip of the nail. Figure 8-Five years after operation. The bony shell is nou well formed; the clear space around the tip of the nail indicates that the nail is moving with the femur.

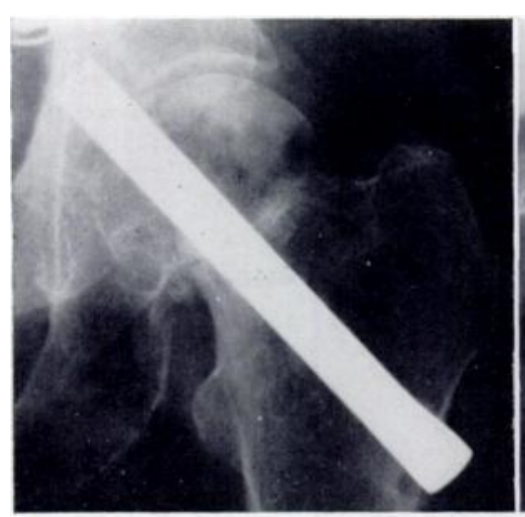

Fig. 9

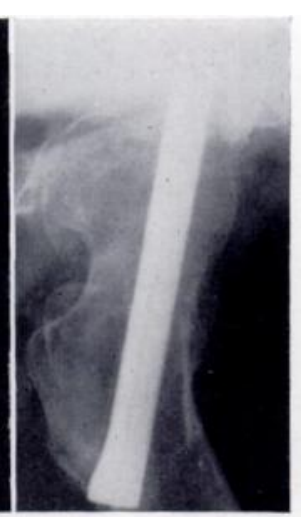

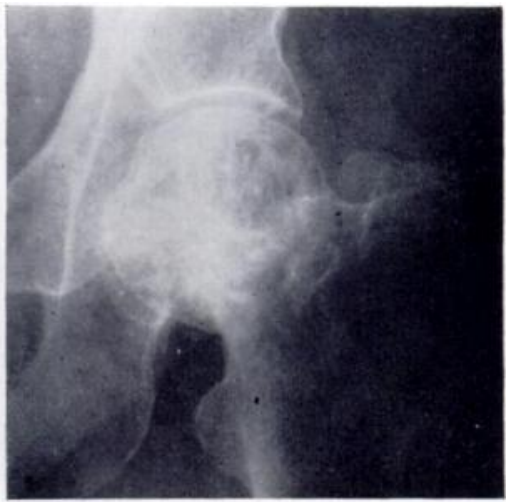

FIG. 10

Erosion of acetabular floor caused by transarticular nailing. Figure 9--Antero-posterior and (right) lateral radiographs, showing condition six months after nailing. The nail is low in the neck and anteriorly placed in the head. There has been good union of the fragments. Note the erosion of the acetabular floor caused by the tip of the nail. Figure 10-Thirty months after injury and twenty months after removal of the nail. There has been no collapse of head although there is some sclerosis in the head, neck and trochanter. The erosion of the acetabular floor persists.

Such a patient often requests to be relieved of the nail after consolidation is complete. After removal of the nail the defect appears to be permanent and does not cause a collapse of the femoral head (Figs. 11 to 13). Sometimes the reactive shell of bone becomes resorbed after removal of the nail (Figs. 14 and 15). These circumstances are quite well tolerated by old people whose activity is reduced both physiologically and even sometimes on advice. 
However, when a joint fixed by a transarticular nail is subjected to shearing forces intolerance is produced. Transarticular nailing is not tolerated in rats subjected to the stress of full weight bearing even without fracture of the neck. Jarry and Uhthoff (unpublished) have shown that in such cases erosion occurs not only in the acetabulum but also along the nail. If a force is applied constantly to mobilise a transfixed joint one of three things can happen: 1) the nail will break, or 2) it will bend, or 3) the bone will yield to pressure. In man, if the force is applied gradually after sixty days the bone yields to pressure and the acetabular wall is eroded. It seems that a cavity by itself, or a defect in a joint, is tolerated, but that a joint locked by a transarticular nail will be tolerated only if the amount of stress imposed on it is controlled.

Factors contributing to stability in transarticular fixation-No disengagement of the nail was observed in the forty-four patients treated. In the one case of pseudarthrosis reduction was in varus and the nail was too eccentric in the head. There were two cases of delayed union.

The action of the nail in transarticular fixation is first to hold the proximal fragment firmly, and secondly to immobilise the joint.

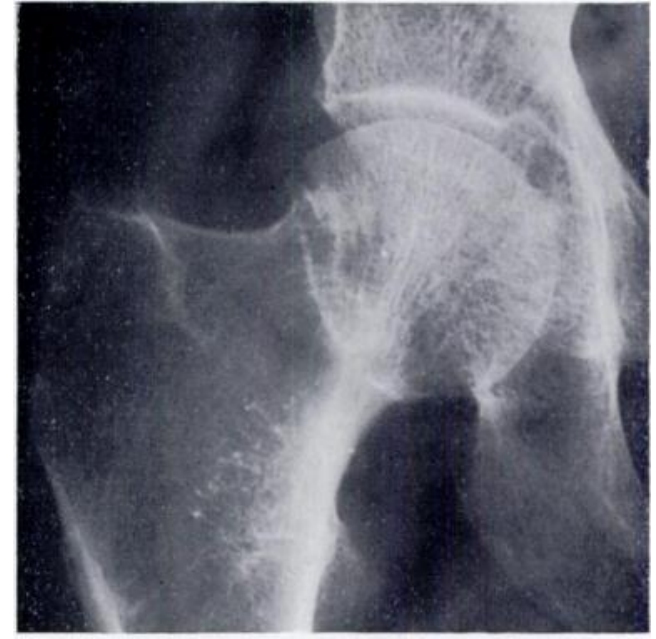

Fig. 11

Persistence of defect in acetabular floor. Radiograph of same hip as that shown in Figures 4 and 5 six years after operation. Note the zone of erosion in the acetabular floor. There is no evidence of arthritis. The patient had normal function of the hip.

By locking the hip joint one adds a proper and adequate immobilisation which is a basic principle in the treatment of every fracture. If this procedure fails to eliminate hip movements completely, at least it reduces their amplitude.

In spite of this more adequate immobilisation the hip is subjected to shearing forces, originating from muscular antalgic contraction or from anti-gravity contraction (Massie 1958). The hip, centre of numerous lever systems, is subjected to extensive shearing forces as demonstrated by Inman (1947). This emphasises the importance of care after operation in the presence of shearing stresses on an active hip undergoing a healing process.

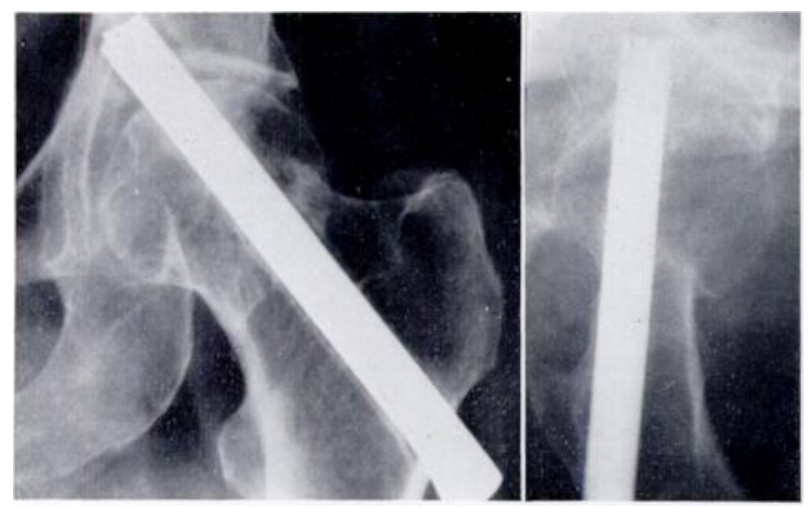

Fig. 12

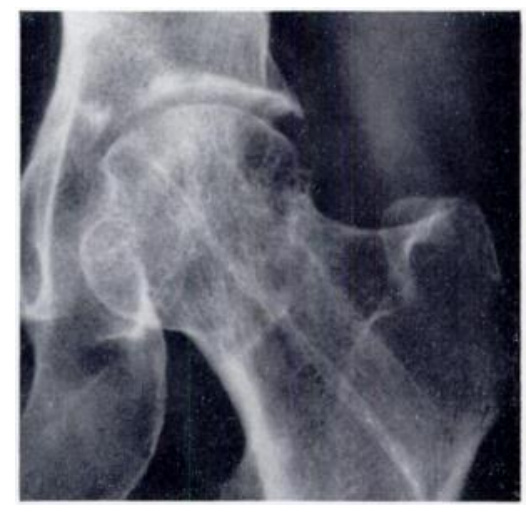

FIG. 13

Persistence of acetabular defect. Figure 12--Antero-posterior and (right) lateral radiographs showing position fourteen months after transarticular nailing. A " tight locking " effect has been produced.

The nail was rather high in the neck head and acetabulum. It was removed in the fifteenth month. Figure 13-Twenty-eight months after operation. Defect in acetabulum still visible, but fracture well united and no collapse of femoral head.

VOL. $46 \mathrm{~B}$, NO. 4, NOVEMBER 1964 


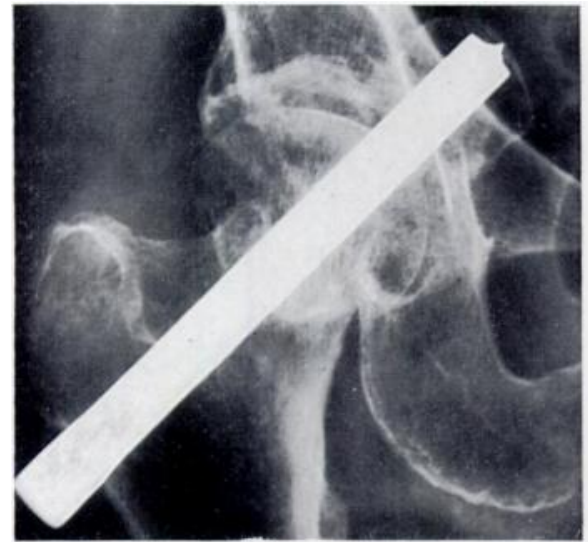

FIG. 14

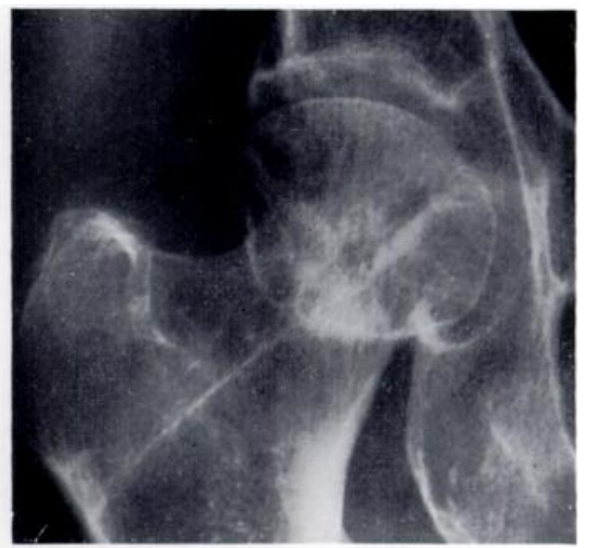

Fig. 15

Resorption of reactive shell of bone. Figure 14--Radiograph showing hip six months after transarticular nailing with "tight locking "effect. Note the shell of bone around the tip of the nail. Figure 15-Fifteen months later, eleven months after removal of nail. The shell of bone is no longer present; the acetabular defect persists, but there is no collapse of the femoral head.

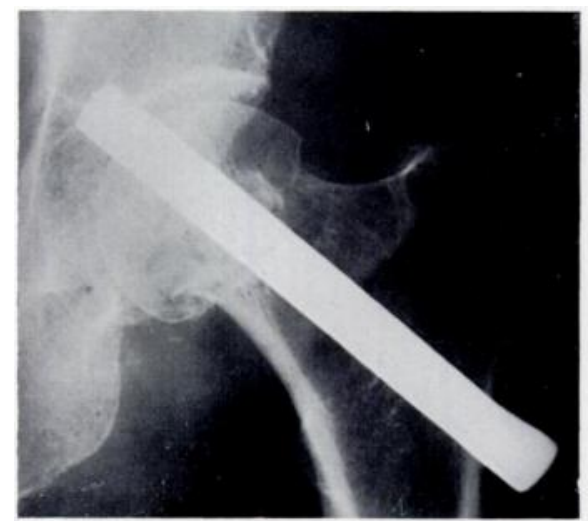

Fig. 16

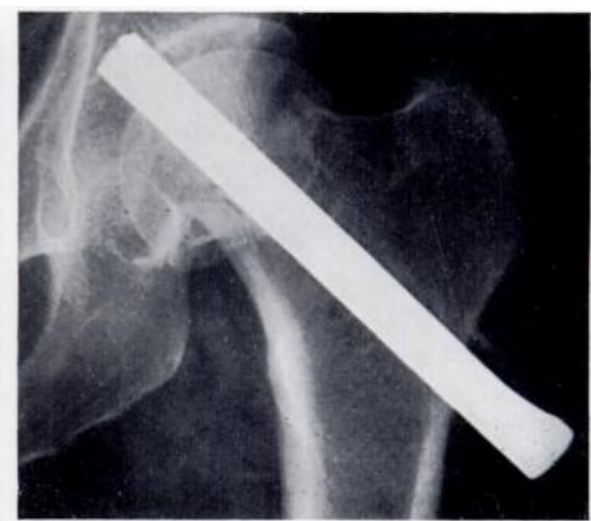

FIG. 17

Impaction of neck into head, associated with osteoporosis. The patient used the wheelchair immediately after operation. Figure 16-Radiograph showing position soon after beginning of mobilisation. Figure 17--Three weeks later. Note the marked impaction of the femoral neck into the head.

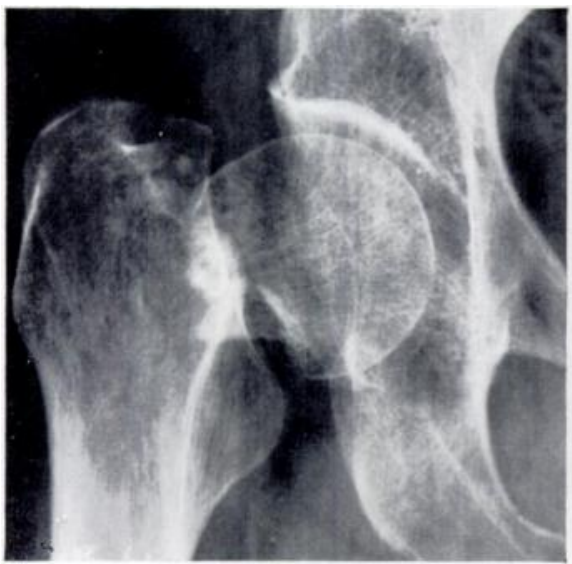

FIG. 18

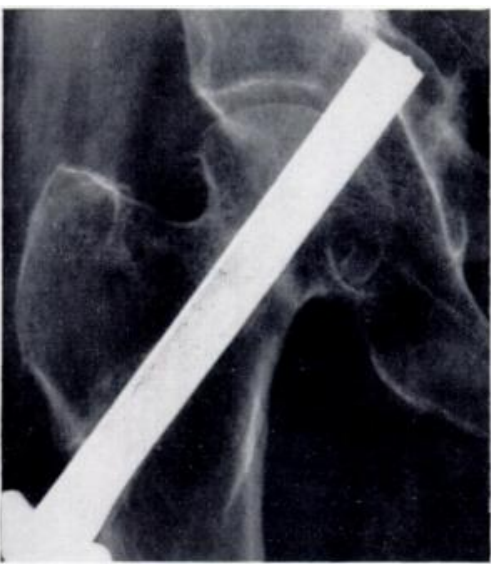

FIG. 19

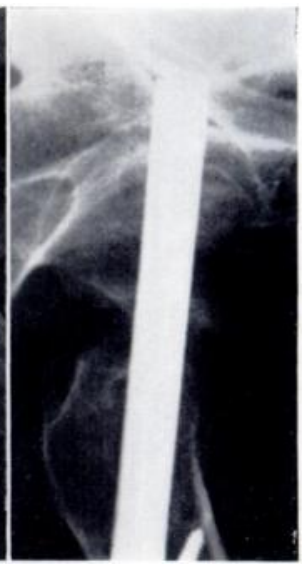

12

Diaphysial fixation for subcapital fracture. Figure 18-Radiograph showing fracture before reduction. Note the evidence of osteoporosis. Figure 19-Antero-posterior and (right) lateral radiographs showing transarticular nailing with diaphysial fixation and "tight locking " effect. Fourteen months after injury the fracture has united. There is evidence of movement of the nail in the pelvis and there is some decalcification around the base of the nail.

THE JOURNAL OF BONE AND JOINT SURGERY 
With the conventional method of fixation, which does not give permanent rigidity, the fulcrum of the lever arm is no longer on a level with the articular cavity, but rather with the fracture line and more precisely with the summit of the nail in the femoral head. At this stage the fulcrum in the head very often works loose, especially in subcapital fractures where the density of the bone is decreased. The cancellous tissue with its vascular elements then erodes.

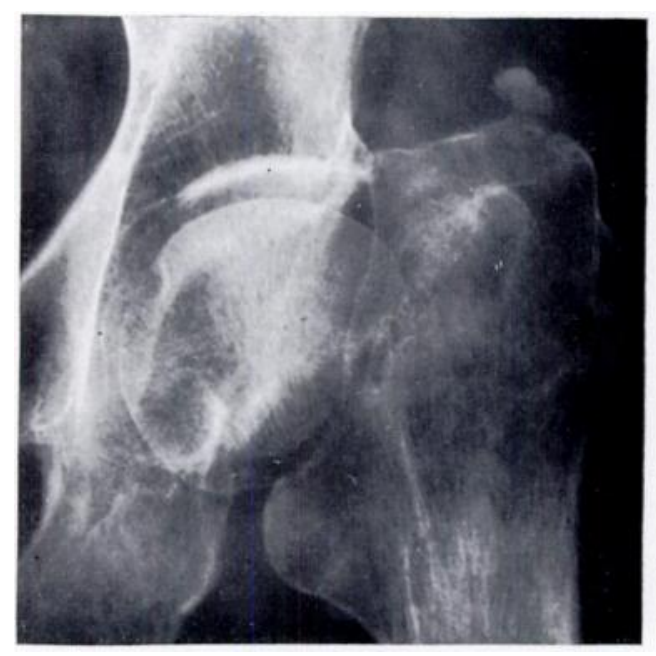

FIG. 20

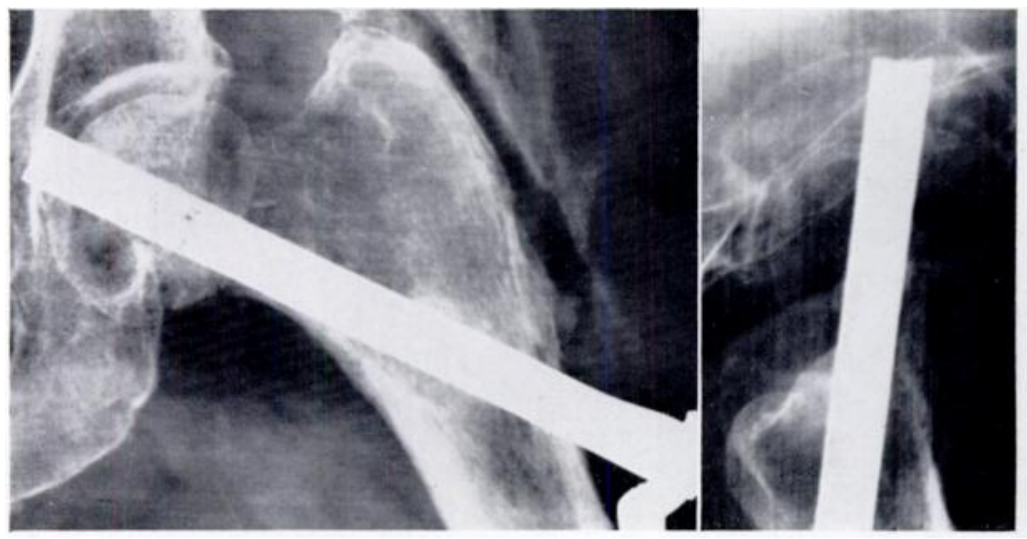

Fig. 21

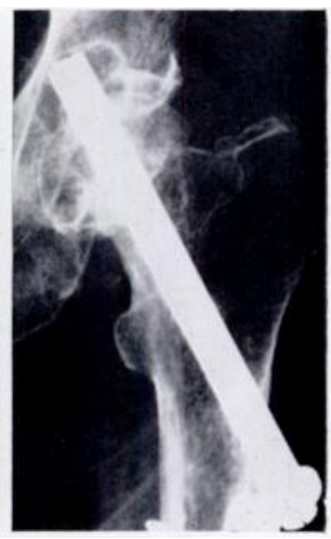

FIG. 22

Damage to weight-bearing area of acetabulum caused by transarticular nailing with diaphysial fixation. Figure 20-Subcapital fracture of femoral neck seventy-nine days after injury. No primary treatment was given. Figure 21-Antero-posterior and (right) lateral radiographs after reduction and transarticular nailing with diaphysial fixation. It was found impossible to reduce the displacement without abduction to 39 degrees. Loose locking effect. Figure 22-Nine months later. There is marked erosion of the weight-hearing part of the acetabulum, caused by the excessive abduction. However, there is no deformity of the femoral head, and the patient had no pain.

In certain cases the erosion is so marked that the nail disengages. On the contrary, if the nail reaches the wall of the acetabulum the proximal fragment is held firmly, and the head of the femur is therefore squeezed between the neck and the acetabulum. Shearing forces cause the neck to be pushed into the head (Figs. 16 and 17) instead of producing disengagement and osteoporosis at the tip of the nail.

We have usually accepted the reduction as shown after the first control radiograph. Moreover, it has been observed, as is generally admitted, that the valgus position of the head

VOL. 46 B, NO. 4, NOVEMBER 1964 
is one factor producing stability and aiding early consolidation. This observation seems to apply to the Pauwels' type 3 (Fig. 3).

Diaphysial fixation was done for four subcapital fractures where reduction was in varus or where the bone was osteoporotic (Figs. 18 and 19). In all but one of the latter cases the penetration of the nail in the acetabulum was always complete. In fact, in one case too wide

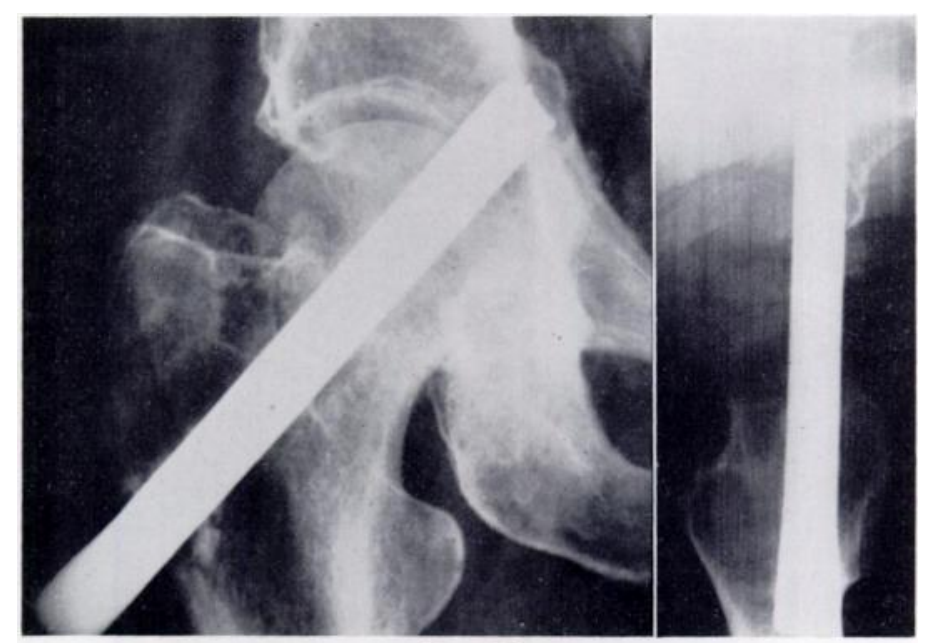

FIG. 23

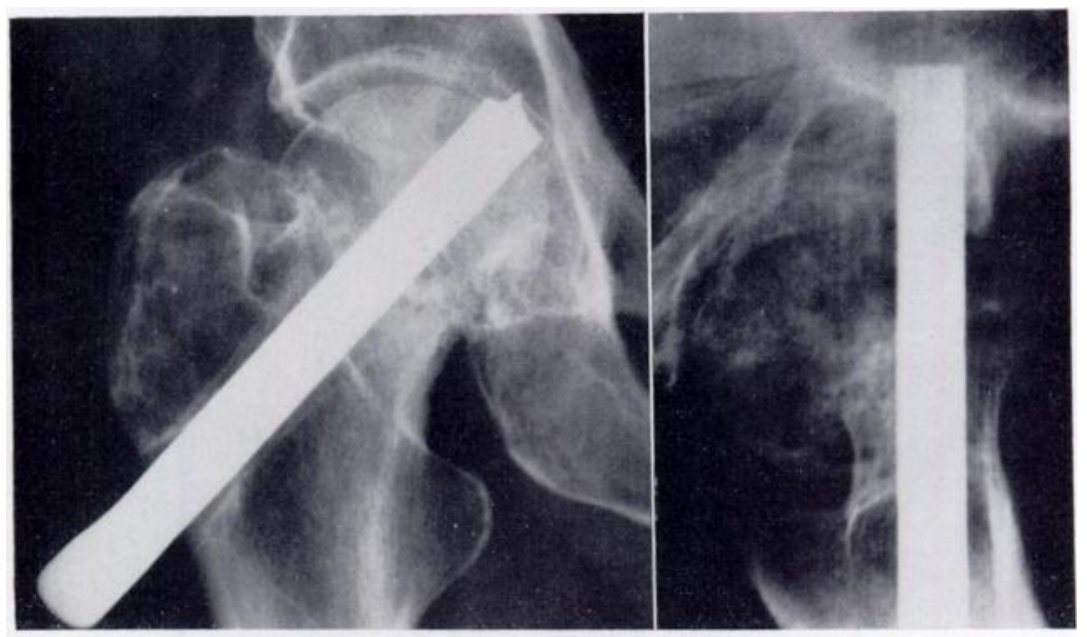

FIG. 24

Delayed union after anterior placing of nail. Figure 23-Antero-posterior and (right) lateral radiographs showing subcapital fracture six months after transarticular nailing with tight locking effect. The nail is anteriorly placed both in the neck and in the head. Figure 24--At seventeen months. There is extrusion of the nail, lateral rotation of the shaft, rotation of the head and delayed union. The patient was active and had no pain.

(39 degrees) abduction caused by technical error with a loose locking effect caused partial destruction of the weight-bearing zone, although the femoral head showed no deterioration (Figs. 20 to 22).

Frankel (1959) showed that the femoral head is the strongest element in the proximal end of the femur as far as supporting osteosynthetic material is concerned. The resistance of the cancellous tissue of the head is estimated at from 2.3 to 3.4 kilograms per square millimetre (Hardinge 1949). It necessarily follows that a nail which completely penetrates the 
head, including the cartilaginous zone, confers far greater stability than one which engages only three-quarters or a half of its thickness.

The question is raised of whether complete penetration of the acetabular wall with a tight locking effect, or a partial penetration with a loose locking effect is preferable. The number of cases operated on does not permit any conclusion. Again, it is debatable which is the most suitable site in the femoral head and neck and in the acetabulum for the introduction of the nail. The few complications that occurred were encountered when the nail was eccentrically placed in the anterior quadrants of the neck and of the head (Figs. 23 and 24). No retroversion of the head was observed when the nail was in the postero-inferior or posterosuperior quadrant of the neck of the femur except in the case of comminution of the back of the femoral neck.

To sum up, it seems that the absence of disengagement and the reduced rate of pseudarthrosis could be explained by an adequate or a permanently rigid immobilisation of the proximal fragment and by the locking of the joint.

\section{COLLAPSE FROM ISCHAEMIC NECROSIS}

In spite of all research during the last ten years, the question of circulation still remains one of the most difficult to assess. Studies on circulation with P32 (Tucker 1950; Boyd, Zilversmit and Calandruccio 1955; Arden 1960) have confirmed that the vascularity of the femoral head varies in relation to time and site. Arden (1960) mentions the discrepancy between expected and actual results: in 42 per cent of hips with a low-rate count the fractures consolidated, and two out of twelve heads with a low or negative P32 ratio did not show avascular necrosis. Harrison (1962) also reports that three out of fifteen viable femoral heads showed signs of necrosis.

Clearly, it must not be taken for granted that the fate of the femoral head is determined at the moment of injury. Again, ischaemia is not the only cause of failure after operation on a broken femoral neck (Arden 1960, Coleman and Compere 1961, Barnes 1962). Further research is essential in order to ascertain all the factors acting on the fate of the head, in particular the action of early mobilisation and shearing stresses on the process of creeping substitution, when the head is subjected to different circulatory gradients and to fixation of different degrees of rigidity.

Limitation of adduction caused by the nail, when the head is in valgus position, may be an important factor in preventing tension in the ligament of the femoral head and its foveolar artery, so preventing ischaemia and avascular necrosis. Certainly an extreme valgus position interferes with blood supply (Smith 1959).

Obviously no definite stand can be taken on a series of ten cases. However, it seems that transarticular fixation at least does not increase the incidence of failure through collapse caused by ischaemia.

\section{SUMMARY AND CONCLUSIONS}

1. A technique of transarticular nailing for subcapital and transcervical fractures of the neck of the femur is described.

2. Forty-four operations have been done, and twenty-one patients have been under observation for periods of from one to six years. In one of these pseudarthrosis has developed; in two others there has been delayed union.

3. The method gives sound fixation by a " squeezing effect " on the proximal fragment, which is enhanced by the locking of the adjacent articulation.

4. It is presented as an alternative to prosthetic replacement for treatment of femoral neck fracture in the aged. The nail damages the articular surface of the acetabulum but not the femoral head.

VOL. 46 B, NO. 4, NOVEMBER 1964 
5. The importance of care after operation is stressed.

6. The advantages of the method, the role of circulatory changes in the femoral head and the mechanics of transarticular fixation are discussed.

I wish to express my sincere thanks to Drs H. Uhthoff, P. Vigneault, A. Gaudet and E. Vicas, whose assistance was greatly appreciated.

\section{REFERENCES}

ARDEN, G. P. (1960): Radioactive Isotopes in Fractures of the Neck of the Femur. Journal of Bone and Joint Surgery, 42-B, 21.

Barnes, R. (1962): The Diagnosis of Ischaemia of the Capital Fragment in Femoral Neck Fractures. Journal of Bone and Joint Surgery, 44-B, 760.

BöHLER, L. (1957): The Treatment of Fractures. Volume 2, p. 1,338. Translated from the thirteenth German edition. New York and London: Grune \& Stratton.

Boyd, H. B., Zilversmit, D. B., and Calandruccio, R. A. (1955): The Use of Radio-active Phosphorus ( $\left.P^{32}\right)$ to Determine the Viability of the Head of the Femur. Journal of Bone and Joint Surgery, 37-A, 260.

Coleman, S. S., and Compere, C. L. (1961): Femoral Neck Fractures. Clinical Orthopaedics, $20,247$.

Crawford, H. B. (1960): Conservative Treatment of Impacted Fractures of the Femoral Neck. Journal of Bone and Joint Surgery, 42-A, 471.

Dickson, J. A. (1953): The " Unsolved" Fracture. Journal of Bone and Joint Surgery, 35-A, 805.

EAtoN, G. O. (1956): Internal Fixation in Displaced Intracapsular Fractures of the Femoral Neck. Journal of Bone and Joint Surgery, 38-A, 23.

Frankel, V. H. (1959): Mechanical Factors for Internal Fixation of the Femoral Neck. Acta Orthopaedica Scandinavica, 29, 21.

Hardinge, M. G. (1949): Determination of the Strength of the Cancellous Bone in the Head and Neck of the Femur. Surgery, Gynecology and Obstetrics, 89, 439.

Harrison, M. H. M. (1962): A Preliminary Report of Vascular Assay in Prognosis of the Fractured Femoral Neck. Journal of Bone and Joint Surgery, 44-B, 858.

InMan, V. T. (1947): Functional Aspects of the Abductor Muscles of the Hip. Journal of Bone and Joint Surgery, 29, 607.

KING, T. (1939): The Closed Operation for Intracapsular Fracture of the Neck of the Femur, Final Results in Recent and Old Cases. British Journal of Surgery, 26, 721.

LeIRA, H. (1946): On behandlingsresultata av larhalsfrakturarsved Trondheim sykehus 1933 til 1941. Nordisk Medicin, 31, 2,084.

Massie, W. K. (1958): Functional Fixation of Femoral Neck Fractures; Telescoping Nail Technic. Clinical Orthopaedics, 12, 230.

McElvenny, R. T. (1957): The Immediate Treatment of Intracapsular Hip Fracture. Clinical Orthopaedics, 10, 289.

SMITH, F. B. (1959): Effects of Rotatory and Valgus Malpositions on Blood Supply to the Femoral Head. Journal of Bone and Joint Surgery, 41-A, 800.

TuCKer, F. R. (1950): The Use of Radioactive Phosphorus in the Diagnosis of Avascular Necrosis of the Femoral Head. Journal of Bone and Joint Surgery, 32-B, 100.

Watson-Jones, Sir R. (1955): Fractures and Joint Injuries. Fourth Edition, p. 707. Edinburgh and London: E. \& S. Livingstone Ltd. 